\title{
Survol des principaux types de lentilles cornéennes chez les enfants aphaques âgés de moins de 5 ans
}

\section{Marie-Eve Corbeil, OD, MSc \\ Amélie Ganivet, OD, MSc \\ Langis Michaud, OD, MSc}

École d'optométrie

Université de Montréal

CP 6128 Succursale Centre-ville

Montréal, Québec

H3C 3J7

\section{Résumé}

L'ajustement en lentille cornéenne est souvent le premier choix pour la correction visuelle des enfants aphaques. Il existe plusieurs types de lentilles qui peuvent être ajustées avec succès pour corriger l'amétropie, stimuler adéquatement le développement visuel, mais également préserver la santé oculaire. Plusieurs facteurs sont déterminants pour le choix du type de lentille. Habituellement, la lentille initialement ajustée est en silicone Élastofilcon A (Silsoft; Bausch \& Lomb, Rochester, NY) avec une migration vers une lentille silicone hydrogel sur mesure avec le temps. Bien que l'ajustement chez les jeunes enfants aphaques présente de nombreux défis, les lentilles cornéennes demeurent souvent l'option de choix de la correction des amétropies après une chirurgie de cataracte congénitale. Un survol des principaux types de lentilles cornéennes disponibles pour les enfants aphaques ainsi que leurs caractéristiques sera présenté.

\section{Abstract}

Contact lenses are often the first choice for visual correction of aphakic children. There are several types of lenses that can successfully be fitted to correct the ametropia, stimulate visual development, and maintain ocular health. Several factors are important for choosing the type of lens. Usually, the first lens fitted is a silicone Élastofilcon A (Silsoft, Bausch \& Lomb, Rochester, NY) with a migration to a custom silicone hydrogel lens over time. Although the fitting in young aphakic children presents many challenges, contact lenses often remain the best option for the correction of refractive errors after congenital cataract surgery. An overview of the main types of contact lenses available for aphakic children and their characteristics will be presented. 
$\mathrm{L}$ e terme cataracte congénitale ou infantile est utilisé pour décrire une opacification significative du cristallin durant la première année de vie. ${ }^{1}$ La cataracte est considérée comme la cause évitable la plus importante de déficit visuel chez l'enfant. ${ }^{2,3}$ La prévalence de la cataracte congénitale est estimée à 1 à 15/ 10000 enfants selon les critères utilisés pour le diagnostic de la cataracte et de la population étudiée. Si la cataracte est dense, centrale et supérieure à $3 \mathrm{~mm}$ de diamètre, si la vue du fond d'œil est impossible, ou si la cataracte est associée au strabisme, un traitement chirurgical s'impose. ${ }^{4}$ Il est préférable que la chirurgie s'effectue le plus tôt possible et surtout à l'intérieur de la période critique du système visuel, soit avant 17 semaines. ${ }^{5}$

Entre 2 jours et 2 semaines après l'opération, l'amétropie secondaire à l'aphakie doit être corrigée pour éviter l'amblyopie qui s'installe rapidement en bas âge. ${ }^{6}$ Présentement, les deux méthodes les plus employées pour la correction visuelle sont le port de lunettes et de lentilles cornéennes., ${ }^{3,7}$ Linsertion d'une lentille intra-oculaire se fait moins fréquemment chez les enfants de moins de 1 an en raison des variations importantes de réfraction qui se produisent durant les premières années de la vie de l'enfant causées par l'élongation axiale de l'œil., $3,8,9$

L'ajustement en lentilles cornéennes est souvent le premier choix pour la correction visuelle des enfants aphaques. ${ }^{710} \mathrm{En}$ effet, non seulement l'aspect esthétique est amélioré, mais aussi le grossissement de l'image, l'aniséikonie, les distorsions périphériques, la restriction du champ visuel avec présence d'un scotome mobile (effet Jack in the box) et le poids sont réduits. ${ }^{10,11}$ Ces aspects sont d'autant plus importants si l'aphakie est unilatérale. De plus, les changements réfractifs secondaires à la croissance sont faciles à modifier en changeant les paramètres des lentilles cornéennes au besoin. Il existe plusieurs types de lentilles qui peuvent être ajustées avec succès chez le jeune enfant. Que ce soit en lentille souple, rigide perméable au gaz, en ou en sclérale, l'objectif sera à la fois de compenser l'amétropie du sujet, afin de stimuler adéquatement le développement visuel, mais également de préserver la santé oculaire par un choix de matériau et de designs permettant une oxygénation adéquate de la cornée. Le présent article sera un survol des principaux types de lentilles cornéennes ajustées chez les enfants aphaques ainsi que de leurs caractéristiques.

\section{AJUSTEMENT DES LENTILLES}

Durant les 18 premiers mois suivant la naissance, une croissance rapide de l'œil se produit. Elle entraine généralement une réduction de l'hypermétropie, une augmentation du diamètre cornéen et une diminution de la courbure cornéenne. Des changements fréquents tant au niveau de la courbure de la lentille, du diamètre ainsi que de la puissance de la lentille devront suivre cette évolution et ce, surtout durant les 2 premières années de vie. ${ }^{10}$

Les mesures de puissance cornéenne au kératomètre sont parfois impossibles à obtenir, surtout pour les moins de 2 ans, donc l'évaluation de base peut devoir être faite sous anesthésie générale.,10,12 Les valeurs moyennes d'études antérieures sont plus fréquemment utilisées comme point de départ. ${ }^{10,12,13}$ Russell et al. ont rapporté une puissance cornéenne au kératomètre à la naissance entre 47,00 D. à 48,50 D. et un aplatissement plus rapide chez les patients aphaques. Le bébé a une puissance cornéenne moyenne à l'ajustement initial de 46,3 D. $\pm 2,8$ D., et à 1 an de 44,6 D. $\pm 2,3$ avec une diminution moyenne de 0,2 D. $\pm 0,2$ D. $/$ mois. $^{12}$

Les puissances dioptriques varient aussi durant les premières années. ${ }^{5,10}$ La puissance moyenne requise en bas âge est plus élevée ( $+25,5$ D. \pm 4 D. à 3 mois) et devient moins convexe avec le temps ( $+17,94 \pm 3,8$ D. à 3 ans d'âge) en raison de la croissance de l'oeil. Ceci représente une variation de 0,23 D. par mois..$^{5}$ Le diamètre cornéen mesure à la naissance en moyenne 10 $\mathrm{mm}$ et atteint sa taille adulte vers l'âge de 2 ans avec un diamètre de $11,7 \mathrm{~mm} .{ }^{10}$

De plus, le monde visuel d'un nourrisson se situe en vision rapprochée. ${ }^{10}$ Puisque les enfants ayant subi une extraction du cristallin n'ont plus la possibilité d'accommoder, une compensation pour la distance rapprochée doit être faite. Une correction de $+2,50$ à $+3,00 \mathrm{D}$. est généralement ajoutée à la prescription en vision de loin pour permettre une vision nette au près. ${ }^{8,13}$ Cette correction devrait être réduite à $+1,00$ ou $+1,50$ entre l'âge de 18 à 24 mois, puisque l'enfant 
s'intéresse davantage à des objets éloignés. Vers l'âge de 3-4 ans, la lentille cornéenne devrait être ajustée pour la vision éloignée et des lunettes avec foyer devraient être prescrites pour corriger la vision de près..$^{10}$

Les rayons ultra-violets (UV) sont associés à une variété de maladies oculaires. ${ }^{14,15}$ L'œil aphaque est potentiellement plus vulnérable aux rayons UV dû à l'absence du cristallin qui les filtre partiellement. Certains types de lentilles offrent la possibilité d'ajouter un filtre UV, mais aucune étude clinique ne confirme qu'il soit requis. ${ }^{12}$

D'autres aspects influencent la réussite du port de lentilles cornéennes. ${ }^{11}$ Tout d'abord, ce sont les parents qui sont responsables de l'insertion et du retrait. Donc l'habileté et la motivation de ceux-ci sont déterminantes. De plus, de nombreuses visites sont requises et viennent s'ajouter aux nombreux rendez-vous en pré et post opération de cataracte. Il arrive aussi que les lentilles se déplacent ou même se perdent puisque les enfants se frottent beaucoup les yeux. En effet, les enfants de moins de 8 ans perdraient en moyenne une lentille tous les 9,2 mois. ${ }^{3}$ Ceci occasionne des coûts supplémentaires qui s'ajoutent au prix initial souvent élevé de ce type de lentille. ${ }^{12}$ Dès que l'enfant est en âge de comprendre, il faut lui expliquer que ceci est un bénéfice pour lui et non une punition. ${ }^{3,11}$ Lorsque la correction est unilatérale, l'avantage de la lentille n'est pas toujours évident pour l'enfant qui s'est habitué à utiliser seulement son meilleur œil.

\section{A JUSTEMENT DES LENTILLES SOUPLES}

Les lentilles souples sont les plus fréquemment utilisées chez les enfants puisqu'elles sont les plus faciles à ajuster et à manipuler. ${ }^{10}$ La courbure cornéenne moyenne du nouveau-né se situe entre 48,50 D. $(6,96 \mathrm{~mm})$ et 47,00 D. (7,18 mm) et s'approche de celle de l'adulte vers l'âge de 3 ans avec 43,25 D. (7,8 mm). ${ }^{10,12}$ L'ajustement initial s'effectue généralement $0,5 \mathrm{~mm}$ plus plat que la courbure cornéenne moyenne. $1^{0}$ Ainsi, pour le nouveau-né, la courbure de départ serait idéalement de 7,4 mm. Des modifications régulières de la courbure des lentilles seront nécessaires dû un aplatissement rapide de la cornée durant les 18 premiers mois de vie. ${ }^{10}$ Le diamètre de la lentille souple est habituellement 2,5-3,0 $\mathrm{mm}$ plus large que le diamètre horizontal de l'iris. Chez le nouveau-né, puisque le diamètre cornéen est en moyenne de $10 \mathrm{~mm}$, le diamètre choisi initialement est généralement $12,5-13,0 \mathrm{~mm}$ et sera modifié avec la croissance. ${ }^{10}$ Une lentille offrant un mouvement trop ample sera considérée trop plate et devra être resserrée. A contrario, une lentille offrant un mouvement limité devra être aplatie afin de favoriser un échange lacrymal adéquat sous sa surface. Finalement, la relation courbure/diamètre peut être influencée par le design spécifique de la lentille et sa puissance. Un diamètre plus grand permet un meilleur centrage et une meilleure stabilité de la lentille cornéenne. Toutefois, il devient parfois difficile pour les parents d'effectuer les manipulations si le diamètre de la lentille est trop grand. ${ }^{10}$

\section{LENTILLES SOUPLES EN SILICONE}

Les enfants aphaques devraient porter leur lentille en port prolongé afin de minimiser les problèmes reliés à la manipulation et de stimuler constamment la vision. Actuellement, il existe une seule lentille approuvée pour le port prolongé 30 jours pour le traitement de l'aphakie pédiatrique. Il s'agit de la lentille en silicone Élastofilcon A (Silsoft; Bausch\& Lomb, Rochester, $\mathrm{NY}) .{ }^{16}$ Elle est la lentille de choix, puisque la perméabilité à l'oxygène (DK) est de $340 \times 10^{-11} \mathrm{~cm}^{2}$ $\mathrm{mL} \mathrm{O2/sec} \mathrm{mL} \mathrm{mm} \mathrm{Hg}$ avec une transmissibilité à l'oxygène (DK/t) de 58 à $0,61 \mathrm{~mm} \cdot{ }^{12}$ Elle est disponible en version pédiatrique entre les puissances de $+23,00$ D. à $+32,00$ D. avec des sauts de $3 \mathrm{D}$. Le diamètre est de 11,3 $\mathrm{mm}$ et 3 courbures sont disponibles : 7,5 $\mathrm{mm}$ (45,00 D.), 7,7 mm. $\left(43,75\right.$ D.) et 7,9 mm (42,75 D.). ${ }^{16}$ La lentille ayant une courbure de 7,5 mm est généralement la première ajustée puisqu'elle se rapproche le plus des valeurs théoriquement obtenues avant l'âge de 18 mois., ${ }^{5,10}$ Une courbure plus plate est nécessaire lorsque l'enfant vieillit : ainsi les courbures de 7,7 mm et 7,9 $\mathrm{mm}$ seront généralement utilisées après l'âge de deux ans. À l'âge de 4 ans, presque tous les patients requièrent la courbure de $7,9 \mathrm{~mm} .{ }^{5,10,12}$ La lentille en Elastofilcon A ne comporte pas que des avantages : son coût élevé d'acquisition et de remplacement peut en limiter l'usage. De plus, le diamètre de la lentille s'avère rapidement trop petit en raison de la croissance de l'œil. Elle ne contient aucun filtre UV. Finalement, le silicone est un composé qui attire beaucoup les lipides des larmes nécessitant un remplacement fréquent, de 2 à 4 fois par année selon les patients. ${ }^{10}$ 


\section{LENTILLES SOUPLES HYDROGEL CONVENTIONNELLES}

Les lentilles cornéennes hydrogel conventionnelles en port quotidien sont disponibles en plusieurs paramètres, ce qui facilite l'ajustement. Le matériau est beaucoup plus résistant aux dépôts lipidiques bien qu'il ait une grande affinité envers les protéines. Ces lentilles peuvent être remplacées sur une base annuelle et il est possible d'ajouter un filtre UV. Le coût des lentilles hydrogel, ainsi que leur remplacement, représente une économie substantielle pour les parents. Par contre, ces lentilles offrent une oxygénation médiocre, à haute puissance convexe, ce qui limite énormément leur attrait. ${ }^{10,17,18}$ En effet, elles peuvent entrainer une néovascularisation, un œdème du stroma et même une dysfonction chronique de l'endothélium. ${ }^{19}$

\section{LENTILLES SOUPLES HYDROGEL À REMPLACEMENT FRÉQUENT}

Il existe peu de lentilles souples hydrogel jetables disponibles dans les corrections fortement convexes. La lentille en Benz G offre des paramètres sur mesure pour cette catégorie de patients. ${ }^{20}$ La lentille en omafilcon A offerte jusqu'à +20 D. avec une courbure de 8,6 mm et un diamètre de $14,2 \mathrm{~mm}$. requiert un remplacement mensuel. ${ }^{21}$ Le fait de remplacer régulièrement les lentilles minimise les risques d'infection et l'accumulation de dépôt. Le problème de la perméabilité à l'oxygène demeure, mais le mouvement plus élevé optimise la libre circulation des larmes sous la surface de la lentille. Ce mouvement permet de compenser, en partie, les risques inhérents d'infections reliés aux débris accumulés sous la surface de la lentille. ${ }^{22}$ Leur courbure de base plus plate et un diamètre plus grand font que l'on réserve ces lentilles aux patients plus âgés, soit souvent à partir de 3 ans.

\section{LES LENTILLES SOUPLES EN SILICONE HYDROGEL}

Des lentilles à remplacement fréquent existent également en silicone hydrogel. Il s'agit à la base d'un matériau hydrophile permettant une augmentation de perméabilité par l'ajout de silicone dans la matrice. Le modulus, soit la rigidité relative de la lentille, est ainsi augmenté, de même que son angle de mouillage, ce qui peut induire une sensation d'inconfort. De plus, cette lentille entraîne parfois de l'irritation et le développement de conjonctivite à papilles géantes suite à l'érosion mécanique de la surface oculaire. Les lentilles sont disponibles dans plusieurs paramètres et permettent même la correction de l'astigmatisme. Toutefois due à l'épaisseur importante de la lentille associée à la prescription élevée, la correction demeure plus précise et facilitée avec l'utilisation d'une lentille sphérique combinée avec le port de lunettes pour corriger l'astigmatisme résiduel. ${ }^{10}$ Elles n'ont pas de filtre UV pour les prescriptions requises pour les enfants aphaques. ${ }^{20}$

\section{LES LENTILLES PERMÉABLES AU GAZ}

Les lentilles perméables au gaz (PAG) peuvent aussi être prescrites chez les enfants de tout âge, à condition que les parents collaborent bien à la pose, au retrait et à leur entretien. Les lentilles PAG offrent de nombreux avantages. En effet, elles ont une perméabilité à l'oxygène nettement supérieure et peuvent être fabriquées dans presque n'importe quels paramètres. Il est possible d'ajouter un filtre UV. L'astigmatisme cornéen, même irrégulier, peut être corrigé ce qui aide grandement lors de cataractes traumatiques impliquant la cornée. ${ }^{10}$ Avec ce type de lentilles les infections et la néovascularisation cornéenne sont beaucoup moins fréquentes.,6,10,19 $\operatorname{Par}$ contre l'adaptation initiale au confort peut être plus difficile. De plus, si l'enfant frotte beaucoup ses yeux, il peut causer de l'irritation, des abrasions cornéennes et même l'éjection des lentilles. ${ }^{10}$

\section{LENTILLES SCLÉRALES}

De nos jours, les lentilles mini-sclérales et les lentilles sclérales peuvent être considérées à tout âge. Leur adaptation est plus complexe mais ceci est largement compensé par la qualité supérieure de la vision obtenue, surtout en présence d'astigmatisme. Les lentilles ne touchent pas la cornée, mais s'appuient plutôt sur une couche de fluide et la conjonctive. Elles procurent un confort comparable aux lentilles souples, tout en offrant les qualités des semi-rigides. La perméabilité à l'oxygène est supérieure aux hydrogels et comparable aux silicones hydrogels si le dégagement est optimal. Le matériau contient un filtre UV. Les dépôts adhèrent rarement au point de nuire à la vision ou au confort du patient. Il s'agit d'une excellente alternative à considérer. ${ }^{23}$ Elles sont toutefois peu utilisées actuellement en pédiatrie dû au coût initial qui est environ 10 fois plus élevé qu'une PAG, ainsi qu'en raison du diamètre plus grand de la lentille qui peut être plus difficile pour les parents au départ à manipuler. ${ }^{10}$ 


\section{SUIVI}

Régulièrement il est nécessaire de modifier l'ajustement du type de lentille cornéenne en fonction de la croissance de l'enfant. Ainsi, l'ajustement avec la lentille en Élastofilcon A généralement utilisée comme essai de départ deviendra une lentille silicone hydrogel sur mesure avec le temps. Le passage se fait habituellement en raison des limites apportées par le diamètre de la lentille en silicone et la présence de dépôts nécessitant un remplacement très fréquent, deux facteurs pouvant contribuer à l'inconfort du patient. Le coût de remplacement du produit justifie aussi le choix des lentilles adoptées., ${ }^{5,10,12}$

Les jeunes patients porteurs de lentilles cornéennes doivent être suivis régulièrement afin d'éviter les effets secondaires. Chez les enfants d'âge scolaire, les études ont prouvé que l'adaptation de lentilles cornéennes était faisable, de façon sécuritaire, et ce, de façon comparable aux patients plus âgés. ${ }^{11}$ L'impact psychologique du port de lentille n'est pas à négliger : meilleure acceptation sociale, plus grande estime de soi, activités physique et sports favorisés, vision globale et qualité de vie améliorées., ${ }^{9,24}$ Même les enfants qui adorent leurs lunettes perçoivent les bénéfices de porter des lentilles cornéennes surtout lorsque les erreurs de réfraction sont telles que celles rencontrées chez les enfants aphaques.

\section{CONCLUSION}

Bien que l'ajustement de lentilles cornéennes chez les jeunes enfants aphaques présente de nombreux défis, elles demeurent souvent l'option de choix de la correction des amétropies après une chirurgie de cataracte congénitale. Le choix des lentilles cornéennes souples pour les enfants aphaques est limité par la disponibilité des paramètres et par la perméabilité à l'oxygène. L'évolution de la disponibilité des matériaux facilite la sélection d'une lentille répondant aux besoins du patient. Il est maintenant possible de stimuler adéquatement le développement visuel tout en préservant la santé oculaire.

\section{BIBLIOGRAPHIE}

1. Francis PJ, Berry V, Bhattacharya SS, Moore AT. The genetics of childhood cataract. J Med Genet 2000;37:481-8.

2. Foster A, Gilbert C, Rahi J. Epidemiology of cataract in childhood: a global perspective. J Cataract Refract Surg 1997;23:601-4.

3. Ma JJ, Morad Y, Mau E, et al. Contact lenses for the treatment of pediatric cataracts. Ophthalmology 2003;110:299-305. doi:10.1016/s0161-6420(02)01557-9.

4. Vasavada AR, N. B. Pediatric cataract surgery. Curr Opin Ophthalmol 2006;17:54-61.

5. de Brabander J, Kok JH, Nuijts RM, Wenniger-Prick LJ. A practical approach to and long-term results of fitting silicone contact lenses in aphakic children after congenital cataract. CLAO J 2002;28:31-5.

6. Saltarelli DP. Hyper oxygen-permeable rigid contact lenses as an alternative for the treatment of pediatric aphakia. Eye Contact Lens 2008;34:84-93. doi:10.1097/ICL.0b013e3181leadaa.

7. Ozbek Z, Durek I, Berk TA. Contact lenses in the correction of childhood aphakia. CLAO J 2002;28:28-30.

8. Collins MJ, Franklin R, Davis BA. Optical considerations in the contact lens correction of infant aphakia. Optom Vis Sci 2002;79:234-40.

9. Walline JJ. Children: an untapped population of contact lens wearers. Contact Lens Spectrum 2002;February.

10. Lindsay RG, Chi JT. Contact lens management of infantile aphakia. Clin Exp Optom 2010;93:3-14.

11. Chia A, J. K, Martin F. Use of contact lenses to correct aphakia in children. Clin Exp Optom 2002;30:252-4.

12. Russell, B, Ward MA, Lynn M, et al. The infant aphakia treatment study contact lens experience: one-year outcomes. Eye Contact Lens 2012;38:234-9. doi:10.1097/ICL.0b013e3182562dc0.

13. Moore BD. Optometric management of congenital cataracts. J Am Optom Assoc 61994;5:719-24

14. Ooi JL, Sharma NS, Papalkar D, et al. Ultraviolet fluorescence photography to detect early sun damage in the eyes of school-aged children. Am J Ophthalmol 2006;141:294-8. doi:10.1016/j.ajo.2005.09.006.

15. Roh S, Weiter JJ. Light damage to the eye. J Fla Med Assoc 1994;81:248-51.

16. Vaughan (ON): Bausch \& Lomb. http://www.bausch $\mathrm{ca} /-/ \mathrm{m} / \mathrm{BL} / \mathrm{Canada} /$ Files/Package\%20Inserts/Vision\%20Care/patient-guides/Silsoft-PIFG.pdf.

17. Brennan NA, Efron N, Weissman BA, Harris MG. Clinical application of the oxygen transmissibility of powered contact lenses. CLAO J 1991;17:169-72.

18. Holden BA, Mertz GW. Critical oxygen levels to avoid corneal edema for daily and extended wear contact lenses. Invest Ophthalmol Vis Sci 1984;25:1161-7.

19. Bruce AS, Brennan NA. Corneal pathophysiology with contact lens wear. Surv Ophthalmol 1990;35:25-58.

20. Lapierre M, Quesnel NM. Répertoire: Les Lentilles cornéennes et les solutions. 2014.

21. CooperVision Canada Corp. Lentilles cornéennes souples (hydrophiles) en Omafilcon A. Remplacement planifié ou Jetables à port quotidien. Richmond Hill (ON): CooperVision Canada Corp. https://coopervision.ca/sites/coopervision.ca/files/Omafilcon_A_Daily_Wear_PI_LF0174A_Rev_3_Sept_2012_(French).pdf.

22. Paugh JR, Stapleton F, Keay L, Ho A. Tear exchange under hydrogel contact lenses: methodological considerations. Invest Ophthalmol Vis Sci 2001;42:2813-20.

23. Pullum KW, Whiting MA, Buckley RJ. Scleral contact lenses: the expanding role. Cornea 2005;24:269-277.

24. Walline JJ, Gaume A, Jones LA, et al. Benefits of contact lens wear for children and teens. Eye Contact Lens 2007;33 :317-21. doi:10.1097/ ICL.0b013e31804f80fb.

25. Walline JJ, Jones LA, Sinnott L, et al. Randomized trial of the effect of contact lens wear on self-perception in children. Optom Vis Sci 2009;86:222-32. doi:10.1097/OPX.0b013e3181971985. 